\title{
Elevated C-reactive protein level predicts lower gastrointestinal tract bleeding
}

\author{
MINORU TOMIZAWA $^{1}$, FUMINOBU SHINOZAKI ${ }^{2}$, RUMIKO HASEGAWA $^{3}$, YOSHINORI SHIRAI ${ }^{3}$, \\ YASUFUMI MOTOYOSHI ${ }^{4}$, TAKAO SUGIYAMA ${ }^{5}$, SHIGENORI YAMAMOTO ${ }^{6}$ and NAOKI ISHIGE $^{7}$ \\ Departments of ${ }^{1}$ Gastroenterology, ${ }^{2}$ Radiology, ${ }^{3}$ Surgery, ${ }^{4}$ Neurology, ${ }^{5}$ Rheumatology, ${ }^{6}$ Pediatrics \\ and ${ }^{7}$ Neurosurgery, National Hospital Organization Shimoshizu Hospital, Yotsukaido, Chiba 284-0003, Japan
}

Received February 25, 2016; Accepted April 8, 2016

DOI: $10.3892 /$ br.2016.654

\begin{abstract}
Lower gastrointestinal (GI) bleeding can be caused by colorectal polyps or cancer. The aim of the present study was to identify blood test variables and medications that can predict lower GI bleeding, which would allow for appropriate colonoscopy. The medical records of patients who underwent colonoscopy from September 2014 to September 2015 were retrospectively analyzed. The selected patients included 278 men (mean age, 67.0 111.5 years) and 249 women (mean age, 69.6 \pm 12.0 years). The diagnosis, medications, and blood test variables were compared between patients with and without bleeding. Logistic regression analysis was performed to determine the factors associated with lower GI bleeding. The presence of colorectal polyp and cancer was associated with lower GI bleeding $(\mathrm{P}=0.0044)$ with an odds ratio of $6.71(\mathrm{P}=0.0148)$. No lower GI bleeding was observed in patients taking non-steroidal anti-inflammatory drugs (NSAIDs), corticosteroids, or anticoagulants. The C-reactive protein (CRP) levels were significantly higher in patients with lower GI bleeding $(\mathrm{P}=0.0227)$. The Hb levels were lower in patients with lower GI bleeding, however this finding was not statistically significant $(\mathrm{P}>0.05)$. No blood test variable was associated with lower GI bleeding. Elevated CRP was associated with lower GI bleeding, while there was no association between the medications and lower GI bleeding.
\end{abstract}

\section{Introduction}

Lower gastrointestinal (GI) bleeding occurs if the bleeding source is located distally to the Treitz ligament (1). It can

Correspondence to: Dr Minoru Tomizawa, Department of Gastroenterology, National Hospital Organization Shimoshizu Hospital, 934-5 Shikawatashi, Yotsukaido, Chiba 284-0003, Japan E-mail: nihminor-cib@umin.ac.jp

Key words: colonoscopy, colorectal cancer, colorectal polyp, C-reactive protein be caused by colorectal polyps, colorectal cancer (CRC), or colonic diverticulum (2) and can be lethal in some patients, particularly the elderly (3). It is diagnosed and treated with colonoscopy $(4,5)$.

Lower GI bleeding is usually chronic and stops spontaneously (6). The identification of the bleeding site is important in its management (7). Lower GI bleeding is usually diagnosed with screening colonoscopy. If the risk factors are known, it is be possible to perform colonoscopy for such patients. The risk factors of patients hospitalized for severe lower GI bleeding include medication with non-steroidal anti-inflammatory drugs (NSAIDs) and anticoagulants (8). It is not clear whether NSAIDs and anticoagulants are related to lower GI bleeding diagnosed using screening colonoscopy. Corticosteroids, used in the treatment of autoimmune disease such as rheumatoid arthritis (9), are associated with bleeding from peptic ulcer (1). However, whether corticosteroids are associated with lower GI bleeding remains to be determined.

Blood test variables are easy to obtain and quantitate. These variables can be useful in distinguishing upper from lower GI bleeding, which can aid the clinical diagnosis (10).

In this study, we analyzed the diagnosis of screening colonoscopy and compared medication intake (i.e., NSAIDs, corticosteroids, and anticoagulants) between patients with or without lower GI bleeding. Blood test variables were also analyzed.

\section{Materials and methods}

Ethics statement. The National Hospital Organization Shimoshizu Hospital Ethics Committee approved this study. This study was not considered a clinical trial because the procedures were performed as part of routine clinical practice; thus, written informed consent for inclusion into the study was not required. Patient records were anonymized and retrospectively analyzed. Written informed consent was obtained from all the patients who underwent colonoscopy.

Patients. The selected patients included 278 men (mean age, $67.0 \pm 11.5$ years) and 249 women (mean age, $69.6 \pm 12.0$ years) who underwent colonoscopy. Twelve patients were diagnosed with lower GI bleeding. The causes of lower GI bleeding of these patients are presented in Table I. 
Table I. Diagnosis of lower GI bleeding.

\begin{tabular}{lc}
\hline Diagnosis & No. of patients \\
\hline CRC & 10 \\
Colorectal polyp & 1 \\
Ulcerative colitis & 1 \\
Total & 12 \\
\hline
\end{tabular}

GI, gastrointestinal; CRC, colorectal cancer.

Study design. The medical records of patients who underwent colonoscopy from September 2014 to September 2015 were retrospectively analyzed.

The patient characteristics included in the analysis were the presence of CRC, polyp, and diverticulitis and the intake of NSAIDs, corticosteroids, or anticoagulants. The presence of CRC and polyp were analyzed because they are major causes of lower GI bleeding (11). Colonic diverticulum is another cause of lower GI bleeding (12).

Colonoscopy. Patients underwent colonoscopy for screening, examination of symptoms, or when the fecal occult blood test was positive. The colonoscopy devices used were CF-Q260 and PCF-Q260AI (Olympus, Tokyo, Japan).

Blood test variables. Blood examination was performed before colonoscopy. The blood test variables analyzed were white blood cell (WBC) and platelet (Plt) counts and hemoglobin (Hb), C-reactive protein (CRP), blood urea nitrogen (BUN), and creatinine $(\mathrm{Cr})$ levels.

Statistical analysis. The Chi-square test was used to compare the diagnosis and medication intake. One-way analysis of variance was used to analyze the blood test variables. A logistic regression analysis was performed to identify the factors associated with lower GI bleeding. $\mathrm{P}<0.05$ was determined to be statistically significant. The JMP 10.0.2 program (SAS Institute, Cary, NC, USA) was used for all the statistical analyses.

\section{Results}

To determine whether a relationship exists between each factor (CRC and polyp, diverticulum, NSAIDs, corticosteroid, and anticoagulants) and lower GI bleeding, the number of subjects of those with and without lower GI bleeding were compared (Table II). The presence of CRC and polyp were related to lower GI bleeding $(\mathrm{P}=0.0044)$. No lower GI bleeding was observed among the patients taking NSAIDs, corticosteroids, or anticoagulants.

To determine whether the blood test variables were related to lower GI bleeding, the variables were compared between the patients with and without lower GI bleeding (Table III). The CRP levels were significantly higher in patients with lower GI bleeding $(\mathrm{P}=0.0227)$. The Hb levels were lower in patients with lower GI bleeding; however, this finding was not statistically significant.
Table II. Characteristics of patients with or without lower GI bleeding.

\begin{tabular}{lcccc}
\hline Variables & Bleeding (-) & Bleeding $(+)$ & Total & P-value \\
\hline Total & 515 & 12 & 527 & \\
Patient no. & $249 / 266$ & $6 / 6$ & $255 / 272$ & 0.8470 \\
(men/women) & & & & \\
CRC, polyp & 219 & 10 & 229 & 0.0044 \\
Diverticulum & 87 & 1 & 88 & 0.3932 \\
NSAIDs & 10 & 0 & 10 & 0.4952 \\
Corticosteroids & 42 & 0 & 42 & 0.1555 \\
Anticoagulants & 12 & 0 & 12 & 0.4546
\end{tabular}

GI, gastrointestinal; CRC, colorectal polyp; diverticulum, colonic diverticulum; NSAIDs, non-steroidal anti-inflammatory drugs; PPI, proton pump inhibitor; H2RA, histamine type-2 receptor antagonist.

Table III. Blood test variables of patients with or without lower GI bleeding.

\begin{tabular}{lrcll}
\hline Variables & & Bleeding (-) & Bleeding (+) & P-value \\
\hline WBC $\left(\times 10^{3}\right)$ & $3.50-8.50$ & $6.556 \pm 3.51$ & $8.56 \pm 4.76$ & 0.1264 \\
$\mathrm{Hb}$ & $13.5-17.0$ & $12.9 \pm 2.1$ & $11.3 \pm 3.5$ & 0.0708 \\
$\mathrm{Plt}$ & $15.0-35.0$ & $23.2 \pm 7.5$ & $26.3 \pm 10.7$ & 0.2683 \\
$\mathrm{CRP}$ & $0.00-0.30$ & $0.51 \pm 0.81$ & $3.13 \pm 4.84$ & 0.0227 \\
$\mathrm{BUN}$ & $8.0-22.0$ & $15.2 \pm 4.8$ & $22.3 \pm 26.2$ & 0.1803 \\
$\mathrm{Cr}$ & $0.40-0.70$ & $0.79 \pm 0.18$ & $1.00 \pm 0.87$ & 0.1609 \\
\hline
\end{tabular}

GI, gastrointestinal; WBC, white blood cell; $\mathrm{Hb}$, hemoglobin; Plt, platelet; CRP, C-reactive protein; BUN, blood urea nitrogen; $\mathrm{Cr}$, creatinine.

Table IV. Logistic regression analysis of factors in lower GI bleeding.

\begin{tabular}{lccl}
\hline Variables & Odds ratio & $\chi^{2}$ & P-value \\
\hline CRC, polyp & 6.71 & 5.94 & 0.0148 \\
Diverticulum & 2.48 & 0.74 & 0.39 \\
NSAIDs & 0.00 & 0.00 & 1.00 \\
Corticosteroids & 0.00 & 0.00 & 0.99 \\
Anticoagulants & 0.00 & 0.00 & 1.00 \\
\hline
\end{tabular}

GI, gastrointestinal; CRC, colorectal cancer; diverticulum, colonic diverticulum; NSAIDs, non-steroidal anti-inflammatory drugs; PPI, proton pump inhibitor; H2RA, histamine type-2 receptor antagonist.

The logistic regression analysis result of the factors involved with lower GI bleeding is shown in Table IV. The presence of CRC and polyp was significantly related to lower GI bleeding, with an odds ratio of $6.71(\mathrm{P}=0.0148)$. The odds ratios of diverticulum and medication intake (i.e., NSAIDs, corticosteroids, or anticoagulants) were not reliable because 
Table V. Logistic regression analysis of blood test variables in lower gastrointestinal bleeding.

\begin{tabular}{lccc}
\hline Variables & Odds ratio & $\chi^{2}$ & P-value \\
\hline WBC & 1.00 & 0.15 & 0.7026 \\
$\mathrm{Hb}$ & 1.56 & 1.86 & 0.1730 \\
$\mathrm{CRP}$ & 0.76 & 0.12 & 0.7305 \\
$\mathrm{Plt}$ & 1.05 & 0.16 & 0.6849 \\
$\mathrm{BUN}$ & 1.04 & 0.03 & 0.8620 \\
$\mathrm{Cr}$ & 0.14 & 0.10 & 0.7524 \\
\hline
\end{tabular}

WBC, white blood cell; Hb, hemoglobin; CRP, C-reactive protein; Plt, platelet; BUN, blood urea nitrogen; $\mathrm{Cr}$, creatinine.

their $\mathrm{P}$-values were not significant $(\mathrm{P}>0.05)$. It was suggested that the presence of CRC and polyp was related to lower GI bleeding. These results were consistent with those of previous studies (11).

The logistic regression analysis result of the blood test variables and lower GI bleeding is shown in Table V. No blood test variables had a P-value $<0.05$. Thus, no blood test variable was related to lower GI bleeding.

\section{Discussion}

CRP is an acute-phase response protein and its serum level is elevated during inflammation (13). In the present study, the CRP levels were higher in patients with lower GI bleeding than those without. Koseoglu et al analyzed the CRP levels of patients admitted to the emergency room for GI bleeding and found that the CRP levels were elevated (14). These results suggested that elevated CRP levels were associated with lower GI bleeding. The mechanism of CRP elevation in these patients is not clear. One hypothesis is that CRP elevation may have been caused by the inflammation around the bleeding area (15). In our study, 10/12 patients with lower GI bleeding had CRC or polyp. In a previous study, abdominal ultrasography findings of patients with elevated CRP levels were analyzed (16). CRC was included in the diagnosis of the patients. Aleksandrova et al analyzed the serum CRP levels of patients with CRC and found that the levels were elevated (17). The abovementioned studies indicated that elevated CRP was associated with CRC. This may be explained by the fact that cancerous tissue can cause inflammation in the affected organ (18).

There are few studies available on the relationship between medication and lower GI bleeding. NSAIDs cause mucosal ulceration and inflammation in the lower GI tract (19). However, it is not well-documented whether the lower GI bleeding is caused by NSAIDs. There are no studies on the relationship between corticosteroids and lower GI bleeding. Anticoagulant intake is a risk factor of bleeding following polypectomy (20). It is not clear whether anticoagulants are related to lower GI bleeding diagnosed using screening colonoscopy. In the present study, no relationship was found between lower GI bleeding and intake of NSAIDs, corticosteroids, or anticoagulants. Owing to the small number of patients, it cannot be safely concluded that the medications had no asscoiation with lower GI bleeding. Nonetheless, care should be taken for the management of patients with lower GI bleeding when they are prescribed with NSAIDs and anticoagulants (21).

One limitation of the present study was the limited number of patients. In this study, no relationship was identified between lower $\mathrm{Hb}$ and lower GI bleeding, which may be due to the small number of patients. Our study did not include patients with bleeding due to vascular ectasia or inflammatory bowel disease (22). In the future studies, more patients are to be recruited to evaluate the relationship between CRP levels and bleeding due to various causes.

In conclusion, an elevated CRP level was related to lower GI bleeding. Elevated CRP can potentially predict lower GI tract bleeding.

\section{References}

1. Gralnek IM, Dumonceau JM, Kuipers EJ, Lanas A, Sanders DS, Kurien M, Rotondano G, Hucl T, Dinis-Ribeiro M, Marmo R, et al: Diagnosis and management of nonvariceal upper gastrointestinal hemorrhage: European Society of Gastrointestinal Endoscopy (ESGE) Guideline. Endoscopy 47: a1-a46, 2015.

2. Marion Y, Lebreton G, Le Pennec V, Hourna E, Viennot S and Alves A: The management of lower gastrointestinal bleeding. J Visc Surg 151: 191-201, 2014.

3. Feinman M and Haut ER: Lower gastrointestinal bleeding. Surg Clin North Am 94: 55-63, 2014.

4. Kim SY, Hyun JJ, Jung SW and Lee SW: Management of non-variceal upper gastrointestinal bleeding. Clin Endosc 45: 220-223, 2012.

5. Lhewa DY and Strate LL: Pros and cons of colonoscopy in management of acute lower gastrointestinal bleeding. World J Gastroenterol 18: 1185-1190, 2012.

6. Jang BI: Lower gastrointestinal bleeding: Is urgent colonoscopy necessary for all hematochezia? Clin Endosc 46: 476-479, 2013.

7. Pfeifer J: Surgical management of lower gastrointestinal bleeding. Eur J Trauma Emerg Surg 37: 365-372, 2011.

8. Lanas Á, Carrera-Lasfuentes P, Arguedas Y, García S, Bujanda L, Calvet X, Ponce J, Perez-Aísa Á, Castro M, Muñoz M, et al: Risk of upper and lower gastrointestinal bleeding in patients taking nonsteroidal anti-inflammatory drugs, antiplatelet agents, or anticoagulants. Clin Gastroenterol Hepatol 13: 906-912.e2, 2015.

9. van der Goes MC, Jacobs JW and Bijlsma JW: The value of glucocorticoid co-therapy in different rheumatic diseases - positive and adverse effects. Arthritis Res Ther 16 (Suppl 2): S2, 2014.

10. Tomizawa M, Shinozaki F, Hasegawa R, Shirai Y, Motoyoshi Y, Sugiyama T, Yamamoto S and Ishige N: Laboratory test variables useful for distinguishing upper from lower gastrointestinal bleeding. World J Gastroenterol 21: 6246-6251, 2015.

11. Dahlerup JF, Eivindson M, Jacobsen BA, Jensen NM, Jørgensen SP, Laursen SB, Rasmussen M and Nathan T: Diagnosis and treatment of unexplained anemia with iron deficiency without overt bleeding. Dan Med J 62: C5072, 2015.

12. Yamada A, Niikura R, Yoshida S, Hirata Y and Koike K: Endoscopic management of colonic diverticular bleeding. Dig Endosc 27: 720-725, 2015.

13. Ansar W and Ghosh S: C-reactive protein and the biology of disease. Immunol Res 56: 131-142, 2013.

14. Koseoglu Z, Ozkan OV, Semerci E, Aslan A, Yetim I, Ucar E, Kuvandik G, Temiz M and Borazan A: The relationship between mortality and inflammation in patients with gastrointestinal bleeding. J Int Med Res 37: 1508-1514, 2009.

15. Tomizawa M, Shinozaki F, Hasegawa R, Togawa A, Shirai Y, Ichiki N, Motoyoshi Y, Sugiyama T, Yamamoto S and Sueishi M: Reduced hemoglobin and increased C-reactive protein are associated with upper gastrointestinal bleeding. World J Gastroenterol 20: 1311-1317, 2014.

16. Tomizawa M, Shinozaki F, Sugiyama T, Yamamoto S, Sueishi M and Yoshida T: Ultrasonography for leukocytosis or elevated C-reactive protein. Hepatogastroenterology 58: 1156-1158, 2011. 
17. Aleksandrova K, Jenab $M$, Boeing $H$, Jansen E, Bueno-de-Mesquita HB, Rinaldi S, Riboli E, Overvad K, Dahm CC, Olsen A, et al: Circulating C-reactive protein concentrations and risks of colon and rectal cancer: A nested case-control study within the European Prospective Investigation into Cancer and Nutrition. Am J Epidemiol 172: 407-418, 2010.

18. Wang X and Huycke MM: Colorectal cancer: Role of commensal bacteria and bystander effects. Gut Microbes 6: 370-376, 2015

19. Sostres C, Gargallo CJ and Lanas A: Nonsteroidal anti-inflammatory drugs and upper and lower gastrointestinal mucosal damage. Arthritis Res Ther 15 (Suppl 3): S3, 2013.
20. Shalman D and Gerson LB: Systematic review with meta-analysis: The risk of gastrointestinal haemorrhage post-polypectomy in patients receiving anti-platelet, anti-coagulant and/or thienopyridine medications. Aliment Pharmacol Ther 42: 949-956, 2015.

21. Hashash JG, Shamseddeen W, Skoury A, Aoun N and Barada K: Gross lower gastrointestinal bleeding in patients on anticoagulant and/or antiplatelet therapy: Endoscopic findings, management, and clinical outcomes. J Clin Gastroenterol 43: 36-42, 2009.

22. Zuccaro G: Epidemiology of lower gastrointestinal bleeding. Best Pract Res Clin Gastroenterol 22: 225-232, 2008. 